\title{
EDITORIAL
}

\section{Haunted house}

\section{The use of ghostwriters to pen scientific papers is nothing new, but if we want to get rid of them, stricter authorship rules may not be sufficient for a successful exorcism.}

A recent report in JAMA $(\mathbf{2 9 9}, 1800-1812,2008)$ claimed that tens of articles authored by Merck about their drug rofecoxib were written by communication firms that provide writing services for companies. In addition, some of these articles were signed by researchers who allegedly had little involvement in the study, but were invited (and remunerated) by Merck to lend their names and prestige to the papers.

The immediate reaction from the media and from some journals was to reprimand the company and the authors for their lack of transparency and unethical behavior. In an editorial accompanying the original report, JAMA called for "drastic action" to eliminate practices of this type.

Regarding authorship, the editorial made one specific recommendation: to require from every author a statement on his or her specific contribution to the paper. Although this seems like a good idea for deterring people from lying if they have nothing to do with a given study, its impact is likely to be limited.

There are two types of ghostwriting: writing a paper for which you receive no author credit (but for which you are probably paid) and authoring a paper to which you contribute no work. The first type of ghostwriting is not illegal and is hardly unethical. If it were illegal, it would not be sufficient to include the name and affiliation of the ghostwriter in the acknowledgements section of the paper, as some have suggested. In addition, asking a researcher to include in the author list someone who was paid for such a service is like asking for the inclusion of all the technicians who participated in the experiments, something that is (and should be) done at the discretion of the principal author.

The second type of ghostwriting is more troublesome, and the idea of author contribution statements is intended to address this problem. However, there is no consensus on what constitutes enough work to earn author status, and a contribution statement will not change this fact. Some scientists sign every paper that comes out of their lab, even if their input was strictly monetary. Others insist on being authors even if they simply supplied a reagent, and still others make it to the author list just thanks to 'valuable discussions'. What difference is there between this last group of authors and the scientists approached by a company to provide comments on a paper? Money is exchanging hands, true, but this should not disqualify someone from being an author unless this person took the money solely in exchange for his or her name, or unless such a transaction is banned by the author's institution. Everything else falls within the imprecise way to define authorship that we alluded to above.

At Nature Publishing Group, we encourage authors to add contribution statements to their work. They are free-form statements in which the phrase "X.Y. provided intellectual input" is perfectly acceptable, as we feel that a strictly intellectual contribution is a legitimate reason to be an author. In the absence of compelling reasons to disqualify those hard-to-quantify 'valuable discussions' as a reason for authorship, a contribution statement will not affect who is listed as author and would not have changed who authored the Merck papers.

This is not to say that ghostwriting should be tolerated, but it does mean that we need to define it more clearly, acknowledging that the issue of authorship is not so simple. More importantly, serious accusations of ghostwriting - that an author took money strictly in exchange for his or her name on a paper-should be handled just like other forms of misconduct: by the author's dean, according to the rules of his or her institution. If institutions don't have specific policies on authorship, drafting them would be a good place to start fighting the ghostwriting problem. Haunted institutions, not journals, are better places for the exorcism of this peculiar type of ghost.

\section{Translational moves}

In this issue of the journal, we introduce some changes to underscore our long-standing interest in translational research.

M ost of the papers we publish are translational, and our News and News \& Views sections also emphasize research of this kind. This month, together with some design changes in both sections, we introduce two columns that may appeal to those of you interested in translational research.

In the Community Corner, we take a recent paper and poll three experts who share their views on the study and discuss the direction in which it moves the field. In the Bench $\leftrightarrow$ Bedside section, we select a field and ask one researcher to identify and discuss a preclinical paper that has translational implications and then ask a second researcher to do the same with a clinical paper that opens questions that need to be answered back in the lab.

In addition, we will continuously publish reviews and perspectives with a translational slant, which you will be able to identify by the tinted strip at the top of the page. We hope that you enjoy these columns and look forward to receiving your feedback. 